\title{
Predictors of long-term compliance with continuous positive airway pressure
}

\author{
Malcolm Kohler, ${ }^{1,2}$ Debbie Smith, ${ }^{2}$ Victoria Tippett, ${ }^{2}$ John R Stradling ${ }^{2}$
}

${ }^{1}$ Sleep Disorders Centre and Pulmonary Division, University Hospital Zurich, Switzerland ${ }^{2}$ Sleep Unit, Oxford Centre for Respiratory Medicine, Churchill Hospital, Oxford, UK

\section{Correspondence to}

Dr Malcolm Kohler, Sleep Disorders Centre and Pulmonary Division, University Hospital of Zurich, Raemistrasse 100, 8091 Zurich, Switzerland: malcolm.k@bluewin.ch

Received 25 January 2010 Accepted 26 June 2010

\author{
ABSTRACT \\ Background There are very few data on objectively \\ assessed long-term compliance with continuous positive \\ airway pressure (CPAP). No single factor has been \\ consistently identified as predictive of continued \\ CPAP use.
}

Methods Adherence to and associations with objective CPAP use were examined in 639 of 3900 patients in whom CPAP treatment was started between 1994 and 2005. Kaplan-Meier survival analyses were used to estimate the proportion of patients still on CPAP. Cox regression models were used to explore the effects of covariates on continued use of CPAP.

Results The median (IQR) follow-up time after initiating CPAP therapy was $3.9(1.5-6.9)$ years and the average use of CPAP was 6.2 (4.5-7.3) h/night. The percentage of patients adherent to CPAP after 5 and 10 years was $81 \%$ and $70 \%$, respectively. Multivariate analysis, including gender, age, neck circumference, Epworth Sleepiness Score, oxygen desaturation index (ODI) and research study participation, indicated that ODI was the only clinical variable independently associated with long-term adherence to CPAP (HR per 1 event $=0.97, p<0.001,95 \% \mathrm{Cl} 0.96$ to 0.98 ). ODI categories were significantly associated with the risk for stopping CPAP in multivariate analysis (using ODI group $0-15 / h$ as reference, HR for ODI group $>15-30 / h=0.68, p=0.100,95 \% \mathrm{Cl} 0.43$ to 1.08 ; for ODI group $>30-60 / h=0.37, p<0.001,95 \% \mathrm{Cl} 0.22$ to 0.60 ; and for ODI group $>60 / h=0.17, p=0.001$, $95 \% \mathrm{Cl} 0.06$ to 0.48 ).

Conclusions The majority of patients with sleep-disordered breathing are using CPAP in the long term and the severity of sleep-disordered breathing rather than sleepiness determines long-term adherence to CPAP therapy.

\section{INTRODUCTION}

It has been estimated that $2-4 \%$ of the adult population in Western countries suffer from moderate to severe obstructive sleep apnoea (OSA) with daytime symptoms, and it is becoming more prevalent as the average body weight of the population rises. ${ }^{1}$ The prevalence of milder or minimally symptomatic forms of OSA among middle-aged adults has been shown to be as high as $26 \%,{ }^{1}$ making OSA one of the most frequent diseases and thus of potential epidemiological interest.

In patients with OSA, excessive daytime sleepiness leads to impaired cognitive function and quality of life and is associated with an increased risk of traffic accidents. ${ }^{23}$ OSA has also been implicated as an important causal factor in the development of cardiovascular disease and appears to increase the risk of fatal and non-fatal cardiovascular events. ${ }^{4}$ OSA can be treated effectively with continuous positive airway pressure (CPAP), but as it is often a lifelong condition, CPAP usually needs to be used for a lifetime.

There are very few studies reporting data on objectively assessed long-term compliance with CPAP; only two studies could be identified which included $>100$ patients and with an average followup time of $>1$ year. ${ }^{7}$ The relatively short followup times and considerable differences in the reported rate of patients who continue to use CPAP in these studies make it difficult to draw definitive conclusions. Data on long-term continuation rates are of particular interest in the UK since, based on National Institute for Health and Clinical Excellence (NICE) guidelines, it has been estimated that the use of CPAP over 14 years is expected to be costneutral within the NHS. ${ }^{8}$ Sensitivity analyses showed that the relative cost-effectiveness of CPAP is largely dependent on adherence rates. More data on adherence and on how to define those patients who are most likely to be adherent to CPAP and thus derive maximal benefit from this therapy are therefore needed.

We have addressed this uncertainty by looking at long-term compliance data of a large group of patients with OSA, taking into consideration potential predictors of compliance such as indices of disease severity and patient-related variables.

\section{METHODS \\ Patients}

Between 1994 and 2005, 3900 patients were started on CPAP at the Oxford Centre for Respiratory Medicine because of sleep-disordered breathing. Their names were stored in a database. During this time interval, 418 of the 3900 patients had participated in research studies conducted at the Oxford Centre for Respiratory Medicine, all of which have been approved by the Oxford research ethics committee. All research patients had proven OSA with excessive subjective daytime sleepiness. Complete datasets with follow-up data were available for 393 research patients. In addition to these research patients, 418 patients who had not participated in a study were randomly selected from the database in order to be able to assess the effect of study participation. The number of patients selected from each year was matched to that of the research patients. The medical notes of 246 non-research patients were available and contained data on CPAP compliance. Therefore, data from 639 patients (a 16.4\% sample of the total population of 3900) were available for analysis (figure 1). 


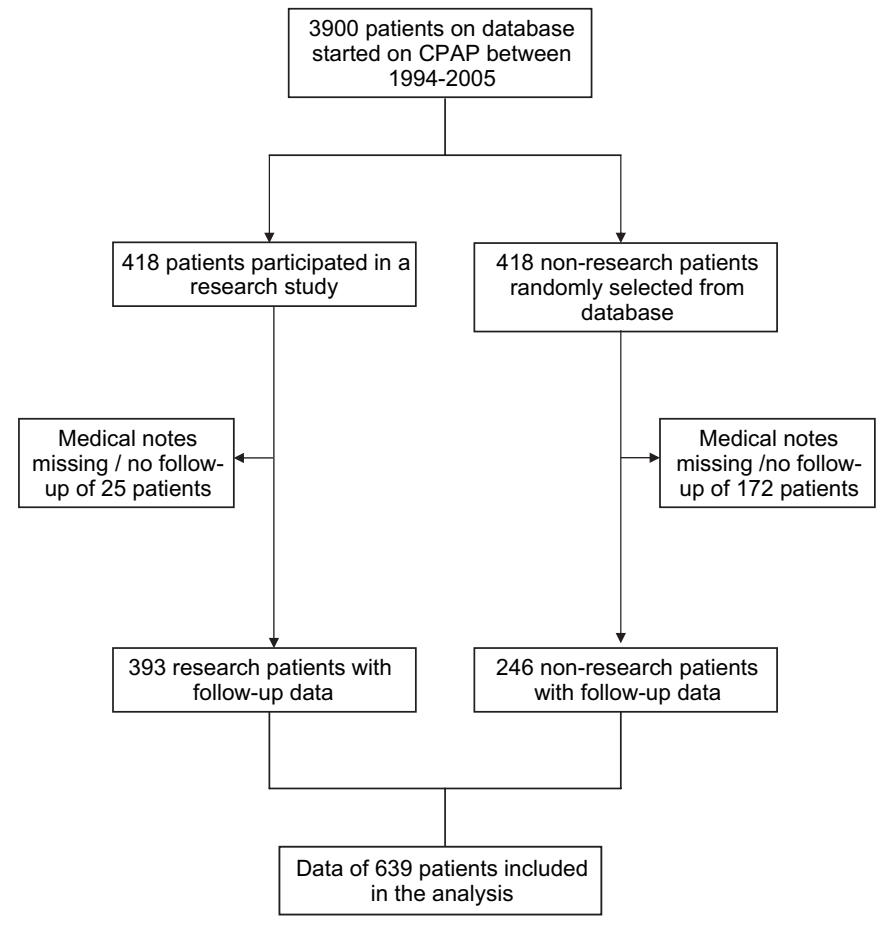

Figure 1 Selection process of the studied population. CPAP, continuous positive airway pressure.

\section{Sleep studies, subjective sleepiness and anthropometrics}

Sleep-disordered breathing was diagnosed from a one night in hospital sleep study. Patients' body movements, heart rate and pulse transit time changes were recorded as measures of arousal from sleep. Pulse oximetry, snoring and increases in the respiratory swing in pulse transit time were used as markers of breathing pattern and respiratory effort (Win-Visi Monitoring System, Stowood Scientific Instruments, Oxford, UK) as previously described and validated. ${ }^{9} 10$ The results of the sleep study were scored automatically, with manual review to ensure accuracy of the data. Sleep-disordered breathing was diagnosed from review of all data and the severity was quantified as the number of oxygen desaturations $>4 \% / \mathrm{h}$ of study (oxygen desaturation index, ODI).

Subjective sleepiness was assessed using the Epworth Sleepiness Score (ESS) which assesses the tendency to fall asleep during eight typical daytime situations. ${ }^{11}$

Data on neck circumference were available for 605 patients and were used as a measure of obesity rather than body mass index because data on body mass index were only available in 413 patients.

\section{Data analysis}

Data are expressed as medians (IOR). All statistical analyses were performed with Statistica V6.0 (StatSoft, Tulsa, Oklahoma, USA). The primary dependent variable of interest was continued use of a CPAP machine. Kaplan-Meier survival analyses were used to allow for variable follow-up times and to estimate the proportion of patients still on CPAP. The log rank test was used to compare rates of continuation of CPAP therapy in four clinical ODI categories $(0-15 / \mathrm{h},>15-30 / \mathrm{h},>30-60 / \mathrm{h}$ and $>60 / \mathrm{h}$ ). In univariate analysis, the association between clinically important factors (including gender, age, obesity, ODI, ESS, CPAP pressure, participation in a research study) and continued use of CPAP treatment was tested using Cox regression analysis. In multivariate analysis, a Cox regression model (including the covariates gender, age, obesity, ODI, ESS and participation in a research study) was used to evaluate independent associations with continued CPAP treatment. Proportional hazard assumptions were tested on the basis of Schoenfeld residuals. A $p$ value $<0.05$ was considered to be statistically significant.

\section{RESULTS \\ Study population}

Data on CPAP compliance were available for 639 patients. The patient characteristics are summarised in table 1 . The median (IOR) follow-up time after initiating CPAP therapy was 3.9 (1.5-6.9) years. The percentage of patients adherent to CPAP therapy after 5 years was $81 \%$, with $70 \%$ after 10 years (figure 2 ). The average use of CPAP at the most recent clinic visit was 6.2 (4.5-7.3) h/night.

\section{Long-term compliance with CPAP}

In univariate analysis the only variables associated with continued CPAP use were ODI, age and current CPAP pressure (table 2). Higher ODI was associated with a considerably higher chance of still using CPAP therapy (figure 3, table 2). Higher CPAP pressure was associated with a slightly higher chance of using CPAP, whereas higher age was associated with a smaller chance of continued CPAP use (table 2). Daytime sleepiness (as assessed by ESS), gender, neck circumference and participation in a research study were not significantly associated with continued CPAP use (table 2).

Multivariate analysis (including gender, age, neck circumference, ESS, ODI and participation in a research study) indicated that ODI event rate but not age remained significantly associated with long-term compliance with CPAP (ODI: HR 0.97, SE $0.01, \mathrm{p}<0.001,95 \%$ CI 0.96 to 0.98 ; age: HR 1.01, SE 0.01 , $\mathrm{p}=0.156,95 \%$ CI 0.99 to $1.03, \mathrm{n}=605)$. Clinical ODI categories were also significantly associated with CPAP continuation in multivariate analysis after correction for covariates (figure 3).

Residual analysis of the models did not detect any deviation from proportional hazard assumptions.

\section{DISCUSSION}

To the best of our knowledge, this study includes data on the longest available follow-up regarding CPAP compliance. In this large study, including more than 600 patients, we found that $81 \%$ of patients with sleep-disordered breathing are using their CPAP machine 5 years after starting the treatment $(n=249)$ and $70 \%$ continue to use it at 10 years $(n=37)$. The average use of CPAP was excellent with $6.2 \mathrm{~h} /$ night. The only clinical factor independently associated with continued CPAP therapy identified was the ODI, but not subjective daytime sleepiness. These

Table 1 Patient characteristics

\begin{tabular}{|c|c|}
\hline & Patients started on CPAP \\
\hline Patients, $\mathrm{n}$ & 639 \\
\hline Age (years) ${ }^{*}$ & $49.5(42.0-57.0)$ \\
\hline $\operatorname{Sex}(M / F)^{*}$ & $594 / 45$ \\
\hline Neck circumference $(\mathrm{cm})^{*}$ & $44.5(43.2-47.0)$ \\
\hline Oxygen desaturation index, events $/ \mathrm{h}^{*}$ & $28.1(14.0-48.4)$ \\
\hline Epworth Sleepiness Score* & $15.0(12.0-18.0)$ \\
\hline Current Epworth Sleepiness Score & $6.0(3.0-8.0)$ \\
\hline Current CPAP pressure $\left(\mathrm{cm} \mathrm{H}_{2} \mathrm{O}\right)$ & $10.2(9.0-12.0)$ \\
\hline
\end{tabular}

Values are medians (IQR) where applicable.

*Values refer to the baseline when CPAP was started.

CPAP, continuous positive airway pressure. 


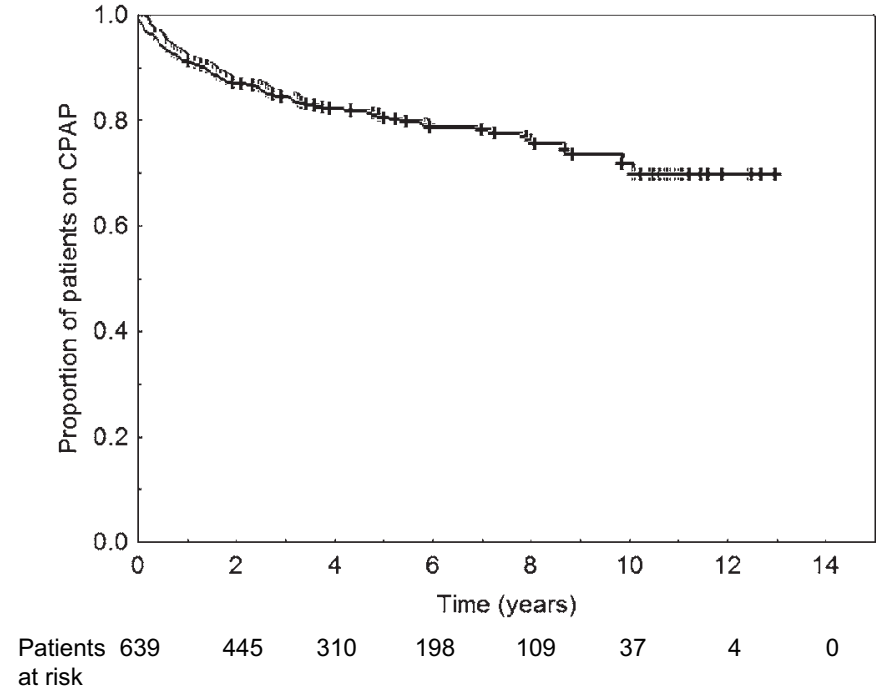

Figure 2 Kaplan-Meier plot showing the proportion of patients using continuous positive airway pressure (CPAP) therapy versus time $(n=639) ; 117$ patients stopped CPAP therapy in 2822 person-years at risk, corresponding to an incidence rate of CPAP termination of 41.5 per 1000 patient-years.

findings imply that the number of sleep-related breathing events rather than daytime symptoms seem to determine long-term adherence to CPAP therapy.

A few studies have attempted objectively to establish adherence rates and predictors of long-term compliance with CPAP therapy. ${ }^{6712}$ In the study by Krieger et al, ${ }^{6}$ which included 608 patients with sleep-disordered breathing, mean follow-up time was 3.2 years; $85 \%$ of the patients were still on CPAP at 7 years and the average CPAP use per night was $5.7 \mathrm{~h}$. McArdle and colleagues $^{7}$ followed 1103 patients with OSA for a median follow-up time of 1.8 years. At 5 years $68 \%$ of the patients were still using CPAP therapy and the mean nightly CPAP use was $5.6 \mathrm{~h}$. Our results extend these previous findings showing that $70 \%$ of the sampled patients who were started on CPAP continue to use it at 10 years. In concordance with the reported nightly CPAP use in the study by Krieger et al and McArdle et $\mathrm{al}^{7}{ }^{7}$ we found an average use of $6.2 \mathrm{~h} /$ night. Although there is no lower threshold known for effectiveness of CPAP, there is some evidence that 3.5-4 h of CPAP use per night are associated with improvements in daytime symptoms, cognitive function and cardiovascular outcomes. ${ }^{13}{ }^{14}$ In our study $83 \%$ of the patients used their CPAP machine for at least $3.5 \mathrm{~h} /$ night, so the vast majority of patients are likely to benefit from the treatment.

Although it has been described previously that the number of apnoeas/hypopnoeas observed in a diagnostic sleep study is a predictor for long-term compliance with CPAP, ${ }^{6}{ }^{7}$ there have been no data available on oxygen desaturations and their

Table 2 Univariate analysis

\begin{tabular}{lllrl}
\hline & HR & SE & p Value & 95\% Cl \\
\hline Gender (male=reference) & 0.80 & 0.30 & 0.553 & 0.39 to 1.65 \\
Age (years) & 1.02 & 0.01 & 0.013 & 1.01 to 1.04 \\
Neck circumference $(\mathrm{cm})$ & 0.96 & 0.03 & 0.152 & 0.92 to 1.01 \\
Oxygen desaturation index (1 event/h) & 0.97 & 0.01 & $<0.001$ & 0.96 to 0.98 \\
Epworth Sleepiness Score & 0.97 & 0.02 & 0.185 & 0.94 to 1.01 \\
CPAP pressure $\left(\mathrm{cm} \mathrm{H}_{2} \mathrm{O}\right)$ & 0.80 & 0.07 & 0.010 & 0.67 to 0.95 \\
Research participation $($ yes=reference) & 1.27 & 0.26 & 0.239 & 0.85 to 1.90 \\
\hline
\end{tabular}

$\mathrm{N}=639$ for all variables except for neck circumference $(n=605)$.

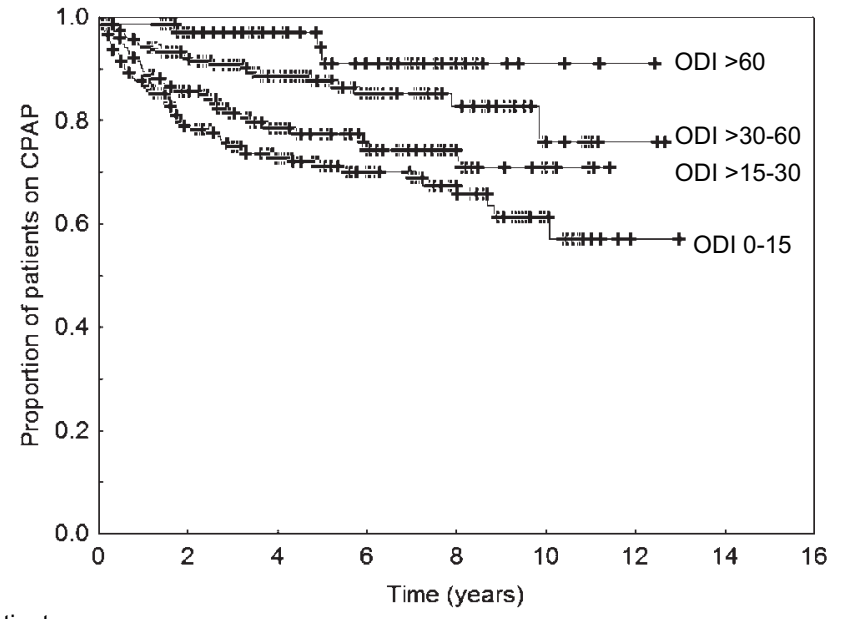

Patients

at risk

$\begin{array}{lcccccccc}\text { ODI } 0-15 & 180 & 122 & 94 & 64 & 41 & 16 & 1 & 0 \\ \text { ODI >15-30 } & 156 & 109 & 71 & 47 & 22 & 10 & 0 & 0 \\ \text { ODI >30-60 } & 220 & 157 & 105 & 62 & 35 & 8 & 2 & 0 \\ \text { ODI >60 } & 83 & 57 & 40 & 25 & 11 & 3 & 1 & 0\end{array}$

Figure 3 Kaplan-Meier plot showing the proportion of patients using continuous positive airway pressure (CPAP) therapy versus time according to oxygen desaturation index (ODI) groups $(n=639)$.

Adherence rates at 10 years were $90.8 \%$ in patients with an ODI of $>60$ / $\mathrm{h}, 77.8 \%$ in those with an ODI $>30-60 / \mathrm{h}, 70.0 \%$ in patients with an ODI $>15-30 / \mathrm{h}$ and $56.9 \%$ in those with an ODI of $0-15 / \mathrm{h}\left(\chi^{2}=25.5\right.$, $\mathrm{p}<0.001)$. ODI categories were significantly associated with the risk of stopping CPAP in multivariate analysis corrected for gender, age, neck size, Epworth Sleepiness Score and research participation (ODI group $0-15=$ reference; ODI group $>15-30, \mathrm{HR}=0.68, \mathrm{p}=0.100,95 \% \mathrm{Cl}$ 0.43 to $1.08 ; \mathrm{ODI}$ group $>30-60, \mathrm{HR}=0.37, \mathrm{p}<0.001,95 \% \mathrm{Cl} 0.22$ to 0.60 ; ODI group $>60, \mathrm{HR}=0.17, \mathrm{p}=0.001,95 \% \mathrm{Cl} 0.06$ to 0.48 ).

potential to predict adherence to CPAP in the long term. We found that ODI showed the strongest association with longterm compliance with CPAP in the univariate analysis and remained significantly associated with continuation of CPAP therapy after correction for covariates in the multivariate analysis. The HR of 0.97 for ODI we found in the multivariate analysis indicates that an increase of 10 in ODI is associated with a risk reduction of $26 \%$ for stopping CPAP. Our results therefore corroborate the findings of previous studies showing that the severity of sleep-disordered breathing seems to be a determinant of long-term adherence to CPAP.

In contrast to other studies, we did not find that subjective daytime sleepiness, as assessed by ESS, is associated with CPAP adherence. $^{67}$ This is an unexpected finding as data from randomised controlled trials suggest that the largest effect sizes with CPAP are observed in patients with severe daytime sleepiness. ${ }^{2}$ Different and more subtle daytime symptoms affecting the patient's quality of life may be more important determinants of long-term compliance with CPAP than the ESS. In this review of our experience in the treatment of a large group of patients with sleep-disordered breathing, we unfortunately have limited information on other daytime symptoms which may influence long-term CPAP adherence. However, in some of the patients with OSA who participated in a research study $(n=302$, age 48.2 (41.0-56.1) years, ODI 34.6 (20.1-53.1), ESS 16 (13.0-18.0), Osler 18.7 (9.8-33.9) $\mathrm{min})$ we do have objective data on daytime sleepiness assessed by the Osler test. ${ }^{15}$ Objectively assessed daytime sleepiness (time awake in the Osler test) in this subgroup was not associated with long-term compliance with 
CPAP in univariate analysis (HR 1.0, $\mathrm{p}=0.35$ ) or in multivariate analysis corrected for gender, age, neck circumference and ODI (HR 1.0, p=0.50), which suggests that the severity of daytime sleepiness at the time of the diagnostic sleep study does not play a pivotal role in terms of long-term treatment adherence. An alternative explanation may be that at our centre we also perform CPAP trials in patients with a relatively low ODI (eg, heavy snorers) but high ESS, where the likelihood of CPAPresponsive disease would be expected to be lower. This would reduce the adherence rate in patients with a high ESS. Conversely, at our centre we also do not put patients with higher ODIs on CPAP if they are truly asymptomatic, so data on CPAP adherence in such patients are not, of course, available.

Similar to the findings in the study by McArdle and colleagues, ${ }^{7}$ age, gender and obesity were not factors associated with long-term CPAP compliance in our study. It therefore appears that these factors should not influence the decision whether or not to treat a patient with CPAP with respect to the expected compliance. This seems especially important in elderly patients who often report little daytime sleepiness, and CPAP treatment might not be offered because of concerns regarding compliance. As the age range in our population was 18-79 years at the time when CPAP was started, our findings are applicable to the vast majority of men seen in an adult sleep clinic.

As the time at which CPAP therapy was started is spread over 11 years in the studied population, there is a theoretical possibility that some factors investigated in our study may have changed over time, thus influencing our results. However, in the last decade there has been no major improvement in the treatment of OSA and no major changes in the way patients with OSA at our institution are approached and treated.

Our study has some limitations; the number of unobtainable medical records was disappointingly high and was due to the repeated moving of the files within the hospital, misfiling and the lack of electronic copies of the medical records. We therefore cannot exclude a potential selection bias as patients with missing files might have been less compliant with CPAP. Furthermore, this was not a prospectively conducted cohort study with clearly defined endpoints, so the impact of some clinical aspects of sleep-disordered breathing (eg, psychological and social factors, humidification, interfaces and short-term CPAP compliance data at 90 days ${ }^{16}$ on long-term CPAP adherence have not been evaluated. Clearly, these issues need to be addressed in further studies.

In conclusion, we have shown in a large population of patients with sleep-disordered breathing that the majority of patients are using their CPAP machine 10 years after starting the treatment. The only independent clinical factor associated with continued CPAP therapy identified was the ODI and not subjective daytime sleepiness. These findings imply that the number of sleep-related breathing events rather than daytime symptoms determines long-term adherence to CPAP therapy in our clinic population. Prospectively conducted cohort studies are needed to further explore potential factors influencing long-term compliance with CPAP.

Funding MK is a recipient of a European Respiratory Society (No 118) and University of Zurich, Switzerland, research fellowship.

Competing interests None.

Ethics approval This study was conducted with the approval of the Oxford research ethics committee.

Provenance and peer review Not commissioned; externally peer reviewed.

\section{REFERENCES}

1. Young T, Peppard PE, Gottlieb DJ. Epidemiology of obstructive sleep apnea: a population health perspective. Am J Respir Crit Care Med 2002;165:1217-39.

2. Siccoli MM, Pepperell JC, Kohler M, et al. Effects of continuous positive airway pressure on quality of life in patients with moderate to severe obstructive sleep apnea: data from a randomized controlled trial. Sleep 2008;31:1551-8.

3. George CF. Reduction in motor vehicle collisions following treatment of sleep apnoea with nasal CPAP. Thorax 2001;56:508-12.

4. Peker Y, Hedner J, Norum J, et al. Increased incidence of cardiovascular disease in middle-aged men with obstructive sleep apnea: a 7-year follow-up. Am J Respir Crit Care Med 2002;166:159-65.

5. Marin JM, Carrizo SJ, Vicente E, et al. Long-term cardiovascular outcomes in men with obstructive sleep apnoea-hypopnoea with or without treatment with continuous positive airway pressure: an observational study. Lancet 2005;365:1046-53.

6. Krieger J, Kurtz D, Petiau C, et al. Long-term compliance with CPAP therapy in obstructive sleep apnea patients and in snorers. Sleep 1996;19:S136-43.

7. McArdle N, Devereux G, Heidarnejad H, et al. Long-term use of CPAP therapy for sleep apnea/hypopnea syndrome. Am J Respir Crit Care Med 1999:159:1108-14.

8. Guest JF, Helter MT, Morga A, et al. Cost-effectiveness of using continuous positive airway pressure in the treatment of severe obstructive sleep apnoea/hypopnoea syndrome in the UK. Thorax 2008;63:860-5.

9. Pitson DJ, Stradling JR. Autonomic markers of arousal during sleep in patients undergoing investigation for obstructive sleep apnoea, their relationship to EEG arousals, respiratory events and subjective sleepiness. J Sleep Res 1998;7:53-60.

10. Pepperell JC, Ramdassingh-Dow S, Crosthwaite N, et al. Ambulatory blood pressure after therapeutic and subtherapeutic nasal continuous positive airway pressure for obstructive sleep apnoea: a randomised parallel trial. Lancet 2002;359:204-10.

11. Johns MW. A new method for measuring daytime sleepiness: the Epworth sleepiness scale. Sleep 1991;14:540-5

12. Meurice J, Dore P, Paquereau J, et al. Predictive factors of long-term compliance with nasal continuous positive airway pressure treatment in sleep apnea syndrome. Chest 1994;105:429-33.

13. Engleman HM, Martin SE, Deary IJ, et al. Effect of continuous positive airway pressure treatment on daytime function in sleep apnoea/hypopnoea syndrome. Lancet 1994;343:572-5.

14. Jelic S, Padeletti M, Kawut SM, et al. Inflammation, oxidative stress, and repair capacity of the vascular endothelium in obstructive sleep apnea. Circulation 2008;117:2270-8.

15. Bennett LS, Stradling JR, Davies RJ. A behavioural test to assess daytime sleepiness in obstructive sleep apnoea. J Sleep Res 1997;6:142-5.

16. Weaver TE, Grunstein RR. Adherence to continuous positive airway pressure therapy. Proc Am Thorac Soc 2008;5:173-8. 